\title{
Recovery of putative pathogens from paper point sampling at different depths of periodontal lesions
}

\author{
Nikola Angelov' \\ Raydolfo M Aprecio' \\ James Kettering' \\ Tord Lundgren ${ }^{2}$ \\ Matt Riggs ${ }^{3}$ \\ Jan Egelberg' \\ 'School of Dentistry, Loma Linda \\ University, Loma Linda, CA, USA; \\ ${ }^{2}$ Department of Periodontology, \\ University of Florida, Gainesville, FL, \\ USA; ${ }^{3}$ Department of Psychology, \\ California State University, San \\ Bernardino, CA, USA
}

\begin{abstract}
Background: The aim of this study was to compare the recovery of three putative periodontal pathogens from periodontal lesions in samples using paper points inserted to different depths of the lesions.

Methods: Twenty 6-8 $\mathrm{mm}$ deep periodontal lesions with bleeding on probing were studied. Microbial samples were obtained using paper points inserted to three different depths of the lesions: orifice of lesion; $2 \mathrm{~mm}$ into the lesion; and to the base of lesion. Culturing was used for recovery and identification of Actinobacillus actinomycetemcomitans, Porphyromonas gingivalis, and Prevotella intermedia.

Results: The recovery of each of the three putative periodontal pathogens was similar following sampling at the various depths of the lesions.

Conclusions: The findings may be explained by the fact that the paper points become saturated as they pass through the orifice of the lesion. Absorption of microorganisms will therefore primarily occur at the orifice. It is also conceivable that the pathogens may be present in similar proportions throughout the various depths of the periodontal lesions.
\end{abstract}

Keywords: paper point sampling, $P$. gingivalis, $P$. intermedia, A. actinomycetemcomitans

\section{Introduction}

Periodontal disease is a multifactoral infection elicited by a complex microbiota in the gingival crevicular area. ${ }^{1-3} \mathrm{~A}$ valid sampling technique is essential to identify and locate the different components of the microbiota of periodontal lesions.

Microbial sampling using endodontic, absorbent paper points inserted to the depth of the periodontal lesion seems to be the most commonly employed sampling method., ${ }^{4,5}$ A concern with this technique is that the samples may not reflect the microbiota of the deeper part of the lesion. The microbiota in the deeper part is conceptually of most interest, since it is close to the area of tissue breakdown. The fact that the paper points are inserted through the orifice might cause them to be saturated by fluids and microorganisms present in the upper part. Reaching the deeper part of the lesion, the paper points may have limited absorptive ability. Thus, a paper point sample might primarily reflect the microbiota of the orifice or the upper part of the lesion.

Baker and colleagues ${ }^{6}$ in an in vitro study used two microbial species and alternated placement of one of them in one of two separate layers in small wells. Paper points were inserted to the bottom of the wells and removed after 10 seconds. Irrespective of which bacteria were placed in the upper or lower layers, more than $90 \%$ of the total colony forming units recovered after culturing originated from the top layer. The authors suggested that this finding may be due to saturation of the paper points as they passed through the top layer.

In a study on periodontal lesions, Smola and colleagues ${ }^{7}$ compared samples taken by paper points with samples using foam tip swabs on the outer surface of the gingiva ('pocket-out' samples). The authors used polymerase chain reaction methods to detect 
five putative periodontal pathogens. No significant difference between the two sampling methods was detected for any of the pathogens. They suggested that both paper point and 'pocket-out' samples represented the microbiota present along the gingival margin.

In addition to sampling technique, the method to identify and quantify the putative periodontal pathogens may have an impact. To date, microbiological culturing has been the mainstay and is still considered to be the 'gold standard' ${ }^{8-10}$

The aim of this investigation was to study if positioning paper points to different depths during bacterial sampling of periodontal lesions has an impact on the recovery of three putative periodontal pathogens, using a culturing technique.

\section{Material and methods}

\section{Subjects}

Twenty patients, 30-60 years of age, to be treated at the Advanced Periodontics Clinic at Loma Linda University School of Dentistry were recruited. Records from initial periodontal examination were reviewed to identify patients having at least one periodontal lesion 6-8 $\mathrm{mm}$ deep with bleeding on probing at the mesio-buccal, mid-buccal, or disto-buccal aspect of a singlerooted tooth. Exclusion criteria consisted of patients: 1) having any systemic disease known to affect periodontal conditions; 2) having any condition for which antibiotic premedication was required; 3) having been on antibiotic treatment within the last three months; and 4) having had periodontal treatment within the last six months. Patients were enrolled in the study after signing an approved informed consent form between January and March 2006. Approval for the study was granted by the Institutional Review Board at Loma Linda University based upon the World Medical Association Declaration of Helsinki.

\section{Clinical procedures}

Following completion of the initial periodontal examination, volunteering and qualifying patients were given verbal and written information about the study and signed an informed consent. The patients returned for bacterial sample collection no sooner than one week later to ensure that the probing procedure would not distort the bacterial samples.

One examiner obtained all samples (author N.A.) using ISO 45 paper points (Dentsply, Tulsa, OK). The tooth to be sampled was isolated with cotton rolls and dried. The supragingival tooth surface was cleaned with a curette and subsequently with cotton rolls. Microbial samples were obtained by inserting points to three different depths of the periodontal lesions:

\section{Orifice of lesion}

The paper point was bent $2 \mathrm{~mm}$ from the tip of the point at an approximate $60^{\circ}$ angle. The bent tip was placed horizontally along the gingival margin of the sampling site and inserted subgingivally until the bent tip was only slightly visible (Figures 1A and 1B). The insertion depth was thus related to the diameter of the paper tip at the $2 \mathrm{~mm}$ level and amounted to no more than $0.3-0.4 \mathrm{~mm}$. The point was removed after 15 seconds, followed by retrieval of a second, similar orifice sample.

\section{Two mm depth}

Two paper points also bent $2 \mathrm{~mm}$ from the tip of the point were inserted at the same sampling site next to each other to this $2 \mathrm{~mm}$ depth and removed after 15 seconds (Figures 2A and 2B).

\section{Base of lesion}

Two paper points were inserted next to each other at the sampling site until resistance was met and removed after 15 seconds.

The sequence of sampling was always orifice level, followed by $2 \mathrm{~mm}$ level, followed by base level (an alternating/rotating sequence was not considered an option due to occurrence of bleeding following sampling the base of the lesion).

\section{Microbiological identification methods}

The two paper points from each sampling depth were pooled in $1 \mathrm{ml}$ of anaerobic dental transport medium (Anaerobe Systems, Morgan Hill, CA) and stored at $-20{ }^{\circ} \mathrm{C}$ until ready to use. The processing of the samples was performed in series of five patients at a time during the week following the sample collection (four weeks total).

For identification of Aggregatibacter actinomycetemcomitans, Brain Heart Infusion agar (Beckton Dickinson and Co, Sparks, MD) and broth with bacitracin and vancomycin (BHI/BV) were used. The BHI/BV agar/broth was
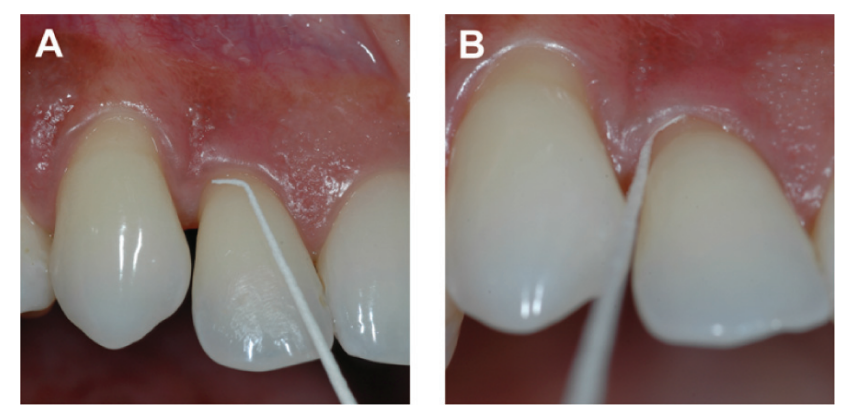

Figure I Paper point bent $2 \mathrm{~mm}$ from the tip of the point prior to insertion (A) and following horizontal subgingival insertion until the bent tip is only slightly visible (B). 

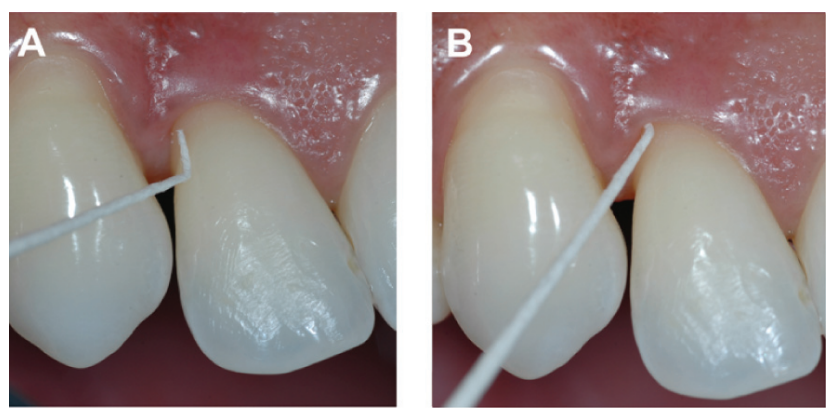

Figure 2 Paper point bent $2 \mathrm{~mm}$ from the tip of the point prior to insertion (A) and following $2 \mathrm{~mm}$ vertical subgingival insertion (B).

prepared freshly every week by adding $75 \mathrm{mg} / \mathrm{L}$ bacitracin (Sigma Chemicals, St Louis, MO), $5 \mu \mathrm{g} / \mathrm{L}$ vancomycin (Sigma Chemicals), $6 \mathrm{mg} / \mathrm{ml}$ yeast extract (Beckton Dickinson and $\mathrm{Co}$ ) and $0.1 \mathrm{ml} / \mathrm{ml}$ horse serum (Hyclone, Logan, UT).

For total anaerobic counts and identification of Porphyromonas gingivalis and Prevotella intermedia, Brain Heart Infusion agar (Beckton Dickinson and Co) and broth with $50 \mathrm{mg} / \mathrm{L}$ hemin (Sigma Chemicals), $15 \mu \mathrm{l} / \mathrm{L}$ vitamin $\mathrm{K} 1$ (Sigma Chemicals), $0.5 \mathrm{~g} / \mathrm{L}$ L-cystein (Sigma Chemicals) and $45.5 \mathrm{ml} / \mathrm{L}$ sheep blood (Hemostat Laboratories, Dixon, CA) was also prepared fresh weekly (BHI/HK).

Prior to analyses of the paper point samples, strains of $A$. actinomycetemcomitans, $P$. gingivalis, and $P$. intermedia were purchased from American Type Culture Collection (ATCC, Manassas, VA) and cultured several times as described below to test the accuracy of the identification and enumeration of the colonies on the agar plates.

For the analyses of the paper point samples, 10-fold serial dilutions with the appropriate broth liquid were made from the samples following thawing and vortexing. One hundred $\mu \mathrm{l}$ of the various dilutions were dispersed onto the surface of agar plates with sterile L-shaped glass rods. The $\mathrm{BHI} / \mathrm{BV}$ plates were incubated in $5 \% \mathrm{CO}_{2}$ at $37{ }^{\circ} \mathrm{C}$ for $2-3$ days. The BHI/ HK plates were incubated in an anaerobic chamber (Coy Laboratory, Grass Lake, MI) at $37^{\circ} \mathrm{C}$ for 5-7 days.

Counts of colony-forming units (CFUs) were performed on the plate dilution that resulted in 30-300 CFUs. If less than 30 CFUs were present at the lowest dilution plated, the number of colonies present was counted and recorded. However, the presence of a single colony was recorded as a 0 value.

For identification of A. actinomycetemcomitans, the plates were flooded with $3 \% \mathrm{H}_{2} \mathrm{O}_{2}$ followed by counts of catalase positive colonies (presence of bubbles on colony) For identification of $P$. gingivalis and $P$. intermedia, longwave UV light (Fisher Scientific, Tustin, CA) and trypsin reaction were used. Dark-pigmented colonies that gave red fluorescence were identified as $P$. intermedia. Then, the plates were slightly misted with $500 \mathrm{mg} / \mathrm{ml}, \mathrm{pH} 7.5$ trypsin reagent (Sigma Chemicals) and re-examined under the UV light. Dark-pigmenting colonies with a blue fluorescence were identified as $P$. gingivalis.

\section{Data analysis}

The recovery of $A$. actinomycetemcomitans at the various levels of sampling was evaluated by comparison of the proportion of positive vs negative samples and by comparison of the CFU counts for positive samples. The recovery of $P$. gingivalis and $P$. intermedia was determined from calculations of the proportions of these microorganisms of the total anaerobic CFU counts. Non-parametric statistics using the Friedman test coupled with Bonferroni corrected post hoc comparisons were used to compare the $\mathrm{CFU}$ counts and the proportions of $P$. gingivalis and $P$. intermedia at the various levels of sampling.

\section{Results}

The recovery of the target microorganisms at the various levels of sampling the periodontal lesions is presented in Table 1. $P$. gingivalis and $P$. intermedia were recovered in all of the 20 patient samples for all the three testing depths. Out of the 20 samples, A. actinomycetemcomitans was found in eight orifice samples, eight $2 \mathrm{~mm}$ samples, and nine base samples. The concordance of positive samples comparing the different sampling levels was as follows: six sample sites were positive for both orifice and $2 \mathrm{~mm}$ level; five sample sites were positive for both orifice and base level; seven sample sites were positive for both $2 \mathrm{~mm}$ and base level; and five samples were positive for all three levels of sampling. The recovery for positive samples ranged between 0.01-5.0 CFUs $\times 10^{3} / \mathrm{ml}$.

Total anaerobic CFUs $\times 10^{5} / \mathrm{ml}$ ranged between 2.3-3.8 for the three sampling levels. Proportions of $P$. gingivalis ranged between $13.2 \%-21.2 \%$. Proportions of $P$. intermedia ranged between $11.0 \%-16.4 \%$ (Table 1 ). The percentage of $P$. gingivalis for the $2 \mathrm{~mm}$ level samples was significantly different from the base samples. No other significant differences of recovery were observed.

\section{Discussion}

In the present study, A. actinomycetemcomitans was recovered from $40 \%-45 \%$ of the samples. This recovery rate is similar to that of previous studies using culturing. ${ }^{11-17}$

$P$. gingivalis and $P$. intermedia were recovered from all samples and in proportions ranging from $11 \%$ to $21 \%$. 
Table I Presence of $P$. gingivalis, $P$. intermedia (proportion of positive samples) and A. actinomycetemcomitans (proportion of positive samples; CFUs $\times 10^{3} / \mathrm{ml}$ for positive samples); total anaerobic CFUs $\times 10^{5} / \mathrm{ml} ; \%$ P. gingivalis; and \% P. intermedia in paper point samples taken at the orifice of the periodontal lesion, points inserted $2 \mathrm{~mm}$ into the lesion, and points inserted to the base of the lesion. Means \pm SD $\mathrm{N}=20$

\begin{tabular}{llll}
\hline & \multicolumn{2}{l}{ Level of sample } & \\
\cline { 2 - 4 } & Orifice & $\mathbf{2 ~} \mathbf{~ m m}$ depth & Base of lesion \\
\hline P. gingivalis & $20 / 20$ & $20 / 20$ & $20 / 20$ \\
P. intermedia & $20 / 20$ & $20 / 20$ & $20 / 20$ \\
A. actinomycetemcomitans & $8 / 20$ & $8 / 20$ & $9 / 20$ \\
A. actinomycetemcomitans CFUs & $0.7 \pm 1.7$ & $0.5 \pm 0.8$ & $0.8 \pm 1.6$ \\
Total anaerobic CFUs & $3.4 \pm 4.3$ & $3.8 \pm 8.2$ & $2.3 \pm 2.4$ \\
\% P. gingivalis & $13.7 \pm 10.5$ & $21.2 \pm 10.3^{*}$ & $13.2 \pm 8.6^{*}$ \\
\% P. intermedia & $15.3 \pm 16.8$ & $16.4 \pm 15.3$ & $11.0 \pm 12.4$ \\
\hline
\end{tabular}

Notes: *Significant difference between $2 \mathrm{~mm}$ and base level samples $(P<0.05)$; The percentage of $P$. intermedia and $P$. gingivalis were determined as proportions of the total anaerobic CFU counts.

Abbreviation: CFUs, colony-forming units.

Recovery of $P$. gingivalis was similar to that reported by some authors using culturing, ${ }^{17-20}$ but higher than that reported by other investigators. ${ }^{13,14,16,21-23}$ Likewise, recovery of $P$. intermedia was similar to that reported by Gajardo and colleagues,${ }^{17}$ but higher than that reported by other investigators using culturing. ${ }^{13-16,21-23}$

The high recovery of $P$. gingivalis and $P$. intermedia in the present study may partly be explained by the fact that deep 6-8 mm lesions with bleeding on probing were sampled. The high recovery may also be explained by the identification method for these microorganisms in the present study. Identification was limited to test of fluorescence under long wave UV light and use of trypsin test. No additional confirmatory tests were employed. Nevertheless, this should not invalidate the findings of the present study, since the same methods were used to compare the recovery at the three different levels of sampling the lesions.

Although statistical difference was detected for the recovery of $P$.gingivalis from $2 \mathrm{~mm}$ depth of sampling, compared to the base of the lesion, the similarity in recovery of the target putative periodontal pathogens for the three different levels of sampling observed in the present study might be explained by two circumstances. As suggested by Baker and colleagues, ${ }^{11}$ the paper points may become saturated as they pass through the orifice of the lesion and the absorption of microorganisms will therefore primarily occur at the orifice. This notion is supported by the findings by Smola and colleagues, ${ }^{12}$ comparing paper point samples with samples using foam tip swabs applied on the outer surface of the gingiva from the gingival margin to the mucogingival junction ('pocket-out' samples). Based upon the similarity in recovery by the two sampling methods, they suggested that both paper point and 'pocket-out' samples may primarily represent the microbiota present along the gingival margin.

It is also conceivable that the pathogens may be present in similar proportions throughout the various depths of the periodontal lesions. If so, this seems to be contrary to the notion that the anaerobic putative periodontal pathogens primarily harbor the deeper parts of the lesions. ${ }^{3,24-27}$

\section{Conclusion}

The findings of the present study seem to cast some doubts about the interpretation of microbial paper point sampling of periodontal lesions.

\section{Disclosure}

Author contributions: Nikola Angelov: patient selection, sample collection, culturing of bacterial samples; Raydolfo M Aprecio: culturing of bacterial samples, technical support; James Kettering: provided laboratory support, design of microbiological methods; Tord Lundgren: manuscript preparation, project support; Matt Riggs: statistical analysis; Jan Egelberg: project idea and design, manuscript preparation. The authors report no conflicts of interest in this work.

\section{References}

1. Zambon JJ. Periodontal diseases: microbial factors. Ann Periodontol. 1996;1:879-925.

2. Holt SC, Ebersole JL. Porphyromonas gingivalis, Treponema denticola and Tannerella forsythia: the "red complex", a prototype polybacterial pathogenic consortium in periodontitis. Periodontol 2000. 2005;38:72-122.

3. Socransky SS, Haffajee AD. Periodontal microbial ecology. Periodontol 2000. 2005;38:135-187.

4. Hartroth B, Seyfahrt I, Conrads G. Sampling of periodontal pathogens by paper points: evaluation of basic parameters. Oral Microbiol Immunol. 1999; 14:326-330. 
5. Tanner AC, Goodson JM. Sampling of microorganisms associated with periodontal disease. Oral Microbiol Immunol. 1986;1:15-22.

6. Baker PJ, Butler R, Wikesjo UM. Bacterial sampling by absorbent paper points. An in vitro study. J Periodontol. 1991;62:142-146.

7. Smola SF, Rettenberger G, Simmet T, Burysek L. Comparison of sample collection methods for the PCR detection of oral anaerobic pathogens. Lett Appl Microbiol. 2003;36:101-105.

8. Zambon JJ, Haraszthy VI. The laboratory diagnosis of periodontal infections. Periodontol 2000. 1995;7:69-82.

9. Loomer PM. Microbiological diagnostic testing in the treatment of periodontal diseases. Periodontol 2000. 2004;34:49-56.

10. Sanz M, Lau L, Herrera D, Morillo JM, Silva A. Methods of detection of Actinobacillus actinomycetemcomitans, Porphyromonas gingivalis, Tannerella forsythensis in periodontal microbiology, with special emphasis on advanced molecular techniques: a review. $J$ Clin Periodontol. 2004;31:1034-1047.

11. Zambon JJ, Christersson LA, Slots J. Actinobacillus actinomycetemcomitans in human periodontal disease. Prevalence in patient groups and distribution of biotypes and serotypes within families. J Periodontol. 1983;54:707-711.

12. Renvert S, Wikström M, Dahlén G, Slots J, Egelberg J. Effect of root debridement on the elimination of Actinobacillus actinomycetemcomitans and Bacteroides gingivalis from periodontal pockets. J Clin Periodontol. 1990;17:345-350.

13. Rodenburg JP, van Winkelhoff AJ, Winkel EG, Goene RJ, Abbas F, de Graff J. Occurrence of Bacteroides gingivalis, Bacteroides intermedius and Actinobacillus actinomycetemcomitans in severe periodontitis in relation to age and treatment history. J Clin Periodontol. 1990;17:392-399.

14. Ali RW, Bakken V, Nilsen R, Skaug N. Comparative detection frequency of 6 putative periodontal pathogens in Sudanese and Norwegian adult periodontitis patients. $J$ Periodontol. 1994;65:1046-1052.

15. Van der Weijden GA, Timmerman MF, Reijerse E, Wolffe GN, Van Winkelhoff AJ, Van der Velden U. The prevalence of A. actinomycetemcomitans, $P$. gingivalis and $P$. intermedia in selected subjects with periodontitis. J Clin Periodontol. 1994;21:583-588.

16. Salari MH, Kadkhoda Z. Rate of cultivable subgingival periodontopathogenic bacteria in chronic periodontitis. J Oral Sci. 2004;46:157-161.
17. Gajardo M, Silva N, Gomez L, et al. Prevalence of periodontopathic bacteria in aggressive periodontitis patients in a Chilean population. J Periodontol. 2005;76:289-294.

18. Loos B, Claffey N, Crigger M. Effects of oral hygiene measures on clinical and microbiological parameters of periodontal disease. J Clin Periodontol. 1988;15:211-216.

19. Loos B, Claffey N, Egelberg J. Clinical and microbiological effects of root debridement in periodontal furcation pockets. J Clin Periodontol. 1988;15:453-463.

20. Boutaga K, van Winkelhoff AJ, Vandenbroucke-Grauls CM, Savelkoul PH. Comparison of real-time PCR and culture for detection of Porphyromonas gingivalis in subgingival plaque samples. J Clin Microbiol. 2003;41:4950-4954.

21. Slots J. Bacterial specificity in adult periodontitis. A summary of recent work. J Clin Periodontol. 1986;13:912-917.

22. Jervoe-Storm PM, Koltzscher M, Falk W, Dorfler A, Jepsen S. Comparison of culture and real-time PCR for detection and quantification of five putative periodontopathogenic bacteria in subgingival plaque samples. J Clin Periodontol. 2005;32:778-783.

23. Rhemrev GE, Timmerman MF, Veldkamp I, Van Winkelhoff AJ, Van der Velden U. Immediate effect of instrumentation on the subgingival microflora in deep inflamed pockets under strict plaque control. J Clin Periodontol. 2006;33:42-48.

24. Kigure T, Saito A, Seida K, Yamada S, Ishihara K, Okuda K. Distribution of Porphyromonas gingivalis and Treponema denticola in human subgingival plaque at different pocket depths examined by immunohistochemical methods. J Periodont Res. 1995;30:332-341.

25. Noiri Y, Shigeyuki E. Identification of periodontal disease-associated bacteria in the "plaque-free zone". J Periodontol. 2000;71:1319-1326.

26. Noiri Y, Li L, Ebisu S. The localization of periodontal disease-associated bacteria in human periodontal pockets. J Dent Res. 2001;80:1930-1934.

27. Noiri Y, Li L, Yoshimura F, Ebisu S. Localization of Porphyromonas gingivalis-carrying fimbriae in situ in human periodontal pockets. J Dent Res. 2004;83:941-945. 
